\title{
Introduction of a partial shift system for house officers in a teaching hospital
}

\author{
D J Vassallo, J Chana, C L Ingham Clark, R E Smith, R F M Wood
}

\begin{abstract}
Objectives-(1) To introduce a partial shift system to reduce the hours of work of preregistration house surgeons to an average of 64 a week to comply with the New Deal for junior doctors; (2) to test linking the partial shift concept to an existing structure of "on call" firms.

Design-Formal assessment after three months of a pilot partial shift system for eight house surgeons on three firms instituted on 1 November 1991, followed by questionnaire and interview evaluation at three and six months of a revised system implemented on 1 February 1992.

Setting-Department of general surgery at St Bartholomew's Hospital, London.

Subjects-24 house surgeons attached to three surgical firms.

Results-In eight weeks each house surgeon worked one week (five shifts) of night duty, one week of "cover" (afternoon and evening) duty, and six weeks of normal daytime hours. Each weekday a house surgeon from the firm on call worked an extended daytime on call shift until $10 \mathrm{pm}$. Weekend duties were split between two house surgeons from the firm on call. A computer generated graphical display of the rota was used to facilitate leave planning. Average working hours were reduced to below 64 per week, including prospective cover, without detriment to patient care and educational standards. Within the shift system individual house surgeons could be on call with their own firm by day and at weekends. Opinions were equally divided among junior staff as to their preference for either on call or partial shift systems.
\end{abstract}

Conclusions-The principles of this partial shift system are generally applicable and the model can readily be adopted by district general hospitals.

\section{Introduction}

The New Deal on junior doctors' hours' is an agreement between the government, NHS management, the royal colleges, and the consultants' and junior doctors' committees of the British Medical Association. It is designed to bring about major reductions in junior doctors' hours of work and improve their overall working conditions while maintaining clinical ànd educational standards. One key proposal is the introduction of partial shift systems in jobs where, in addition to an emergency workload, there is a major commitment to daytime elective work. Doctors in a partial shift system work normal weekday hours most of the time but at intervals work a week of night shifts. The average contracted hours in this system must not exceed 64 per week, including prospective cover.

Shift systems have worked successfully in single units $^{2}$ but we wished to assess whether a satisfactory scheme could be developed to incorporate three separate surgical firms. A pilot partial shift system was introduced for all house officers in general surgery at $\mathrm{St}$ Bartholomew's Hospital on 1 November 1991, shortly after the New Deal was published. The problems exposed by the pilot study resulted in many modifications, and a much improved partial shift system was introduced on 1 February 1992. This paper describes the workings of the system up to 31 July 1992.

\section{Subjects and methods}

Throughout the study there were roughly 80 general surgical patients daily at St Bartholomew's Hospital, mainly accommodated in 50 beds on two adult wards. The remaining patients were in the five day ward, the day surgery unit, the intensive care unit, the children's ward, the renal transplant unit, and the amputee rehabilitation unit. There were three surgical firms, identified by colour codes as Yellow, Green, and Dark Blue. Each firm had a specialist interest, but all covered emergency general surgery. The firms worked a one in three on call rota. Each firm had two consultants, one senior registrar, and a senior house officer. There were eight preregistration house surgeons (three on the Blue firm, three on the Green firm, and two on the Yellow firm). The posts were of three months' duration. The other three months were spent in orthopaedics, urology, or otolaryngology, where the house surgeons were on conventional on call rotas. The general surgical house officers also cared for 20 radiotherapy inpatients and any radiotherapy emergency admissions.

Eight house surgeons participated in the pilot scheme and eight participated in each three months of the revised partial shift system. All house surgeons had previously worked on conventional on call rotas.

\section{PREVIOLS ON CALI ROTA}

House surgeons were previously on a conventional one in four on call rota with prospective cover and contracted for an average of 88 hours per week. The working week varied from 64 to 112 hours, the longest period of continuous duty being 57 hours. There was always one house surgeon on call with his or her own firm, with a second houseman "on cover."

\section{PILOT SCHEME}

The pilot scheme was a compromise between the previous on call rota and a true partial shift system, attempting to maintain an on call and an on cover house surgeon at all times and with weekend duties of 51 hours. Thus two house surgeons worked a night shift for two consecutive weeks (Monday to Friday), alternating first on call and second on call duties each week. After midnight all calls were directed to the first person on call, the second person on call being called only if there were problems. This allowed us to 
monitor the requirement for a second on call house surgeon. The other six house surgeons worked a regular daytime shift ( 745 am till $6 \mathrm{pm}$ ), with an evening extension to $10 \mathrm{pm}$ once per week. This shift did not necessarily coincide with the days their firm was on call. Weekend duties ( 9 am Saturday till 12 noon Monday) followed a set rotation on a two in eight basis. This meant that occasionally neither of the two house surgeons on duty would belong to the firm on call.

Evaluation of pilot scheme-The pilot scheme was assessed by questionnaire and individual consultation by one of us (DJV) with the ward sisters and every member of the surgical and radiotherapy consultant and junior staff. Approved amendments were then incorporated into a revised partial shift system, which was implemented on 1 February 1992.

\section{REVISED PARTIAL SHIFT SYSTEM}

With the revised partial shift system (fig 1) each house surgeon worked only one week of night duty

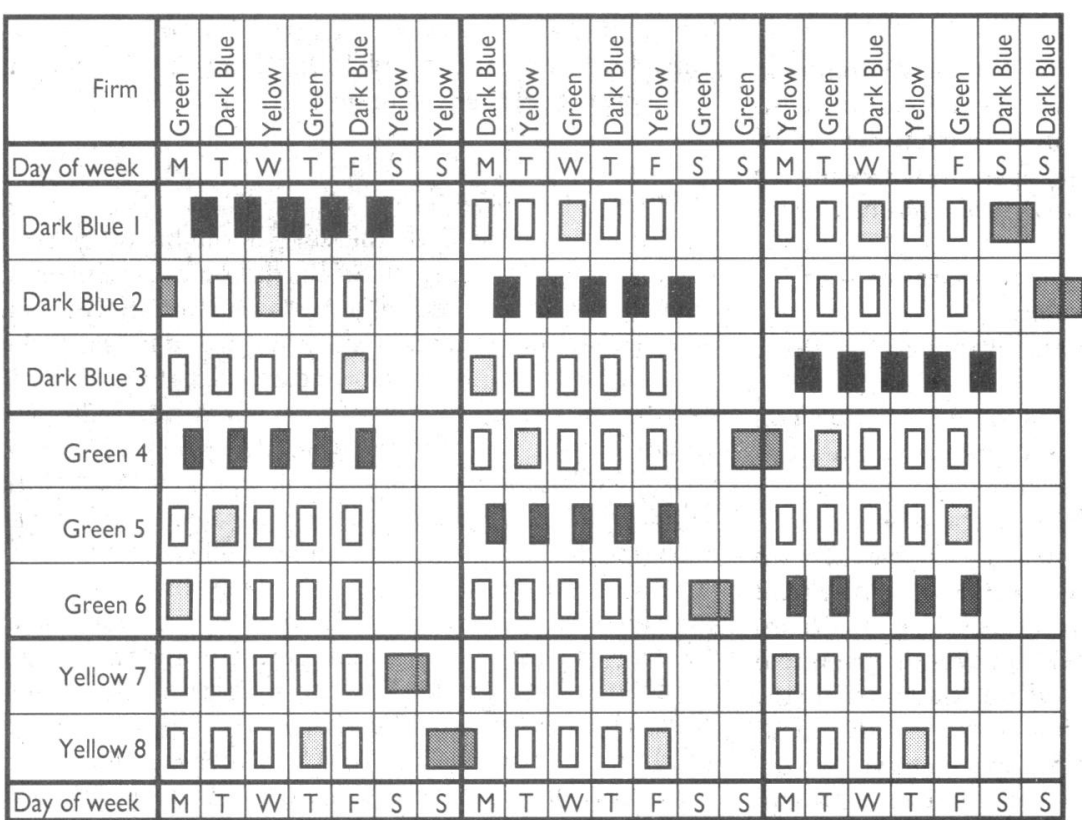

FIG 1-Night and cover shifts in revised partial shift system, February to fuly 1992

\begin{tabular}{|c|c|c|c|c|c|c|c|c|c|c|c|c|c|c|c|c|}
\hline (Month) & \multicolumn{4}{|c|}{ Week I } & \multicolumn{6}{|c|}{ Week 2} & \multicolumn{6}{|c|}{ Week 3} \\
\hline Firm & \begin{tabular}{|l|l|}
$B$ & $A$ \\
\end{tabular} & \begin{tabular}{l|l}
$C$ & $B$ \\
\end{tabular} & A & \begin{tabular}{l|l|}
$c$ & $c$ \\
\end{tabular} & A & $c$ & \begin{tabular}{|l|l}
$B$ & \\
\end{tabular} & \begin{tabular}{l|l}
$\mathrm{A}$ & $\mathrm{C}$ \\
\end{tabular} & & B & C & $B$ & $\mathrm{~A}$ & \begin{tabular}{l|l}
$C$ & $B$ \\
\end{tabular} & & \begin{tabular}{l|l}
$A$ & $A$ \\
\end{tabular} \\
\hline Day of week & \begin{tabular}{|l|l}
$M$ & $T$ \\
\end{tabular} & $\begin{array}{ll}W \\
W\end{array}$ & $F$ & \begin{tabular}{l|l}
$\mathrm{S}$ & $\mathrm{S}$ \\
\end{tabular} & M & $T$ & $w$ & $T \quad F$ & $=\mathrm{s}$ & $\mathrm{s}$ & M & $T$ & W & $T \mathrm{~F}$ & & $\begin{array}{l}\mathrm{s} \\
\mathrm{s}\end{array}$ \\
\hline A I & 1 & 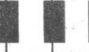 & 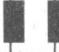 & & & $\square$ & (口) [ & (प) & & & & (口) & & {$[\square$} & & 1 \\
\hline$A 2$ & $\square \square$ & $\square$ & 0 & & & I & 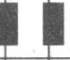 & 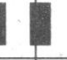 & & & (口) & $\square$ & & $\square[$ & & \\
\hline A 3 & $\square \square$ & 01 & 0 & & (口 & 0 & $0 \mid$ & 口। & & & & 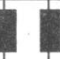 & 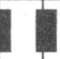 & 1 & & \\
\hline B 4 & $\square \square$ & 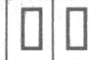 & 1 & & $\square$ & $\square$ & ㅁ] & $0 \mid 0$ & & [ & & 口 & $\square[$ & $\square[$ & & \\
\hline B 5 & $\square$ & (1) & 1 & & $\square$ & $\square$ & 0 & $0 \mid 0$ & {[} & 7 & & $\square$ & $\square[$ & $\square[$ & & \\
\hline $\mathrm{C} 6$ & $\square$ & 미 & $\square$ & & 9 & 0 & 0 & 0 & & & & $\square$ & $\square[$ & $\square[$ & & \\
\hline $\mathrm{C7}$ & $\square$ & $\square$ & $\square$ & प & $\square$ & D & 0 & (] [ & & & & $\square$ & $\square[$ & $\square[$ & & \\
\hline Day of week & \begin{tabular}{|l|l}
$\mathrm{M}$ & $\mathrm{T}$ \\
\end{tabular} & \begin{tabular}{l|l}
$\mathrm{W}$ & $\mathrm{T}$ \\
\end{tabular} & $F$ & \begin{tabular}{l|l}
$s$ & $s$ \\
\end{tabular} & M & $1 \mathrm{~T}$ & $|w|$ & \begin{tabular}{l|l}
$T$ & $F$ \\
\end{tabular} & $\mathrm{~F}$ & 5 & M & $\mathrm{T}$ & $\mathrm{W}$ & \begin{tabular}{l|l} 
& $F$ \\
\end{tabular} & & \begin{tabular}{l|l}
$S$ & $S$ \\
\end{tabular} \\
\hline & (口) & $=745$ & am tc & $\mathrm{pm}$ & & & $\begin{array}{r}=830 \\
\quad \text { (wee }\end{array}$ & $\begin{array}{l}0 \text { am to } \\
\text { ekend) }\end{array}$ & & & & & & & & \\
\hline & $\square$ & $\begin{array}{r}=745 \\
\text { (call) }\end{array}$ & & o $10 \mathrm{pm}$ & & & $\begin{array}{r}=9 \mathrm{pr} \\
\text { (nig }\end{array}$ & $\begin{array}{l}m \text { to } 9 \\
\text { ht) }\end{array}$ & & & & & & & & \\
\hline & $\square$ & $\begin{array}{r}=9 \\
(\mathrm{w}\end{array}$ & & & & & $\begin{array}{r}=2 \mathrm{pr} \\
\text { (co) }\end{array}$ & $\begin{array}{l}m \text { till } n \\
\text { ver) }\end{array}$ & & & & & & & & \\
\hline
\end{tabular}

FIG 2-New partial shift system without cover shift, weeks $1-3$ (five shifts of $9 \mathrm{pm}$ to $9 \mathrm{am}$, or $10 \mathrm{am}$ on Saturdays) and one week of cover ( $2 \mathrm{pm}$ till midnight, Monday to Friday) every eight weeks. In addition to working on his or her own firm, the cover individual looked after radiotherapy patients and, specifically, crosscovered for colleagues on leave or on night duty. The other six weeks were normal daytime hours (7 45 am till $6 \mathrm{pm}$ ). To adapt to the one in three on call commitment of each firm a house surgeon of the firm on call worked an extended on call shift from 745 am till $10 \mathrm{pm}$. Weekend duties were split between two house surgeons of the firm on call ( 9 am Saturday till 9 am Sunday, or 830 am Sunday till 830 am Monday). This was effectively the same as working a day and a night shift consecutively and was within the constraints of the hours controls of the New Deal.

At shift changeover times there was a formal written handover at a designated place. This concentrated on ill patients requiring extra attention, emergency admissions, and elective weekend admissions of other firms.

The working week varied between 53 and 81 hours, including time for handovers and a two hour allowance per week for prospective cover. The average hours worked per week, calculated over the 13 weeks of attachment, varied between 62 and 64 per individual. The house surgeons were therefore contracted for 40 standard hours paid at the $100 \%$ rate and 24 additional duty hours paid at the $70 \%$ rate. ${ }^{3+}$

Before implementing the revised scheme on 1 February 1992 we produced a graphical display of the partial shift system for the next six months. This was constructed with facilities (columns and tables, graphics, and macros) of the wordprocessing package WordPerfect 5.1. This simplified leave planning, which is essential for smooth running of the system: a doctor cannot go on leave when on night duty or when a colleague on the firm is on night duty or on leave.

From 1 August 1992 one house surgeon post will be abolished, together with the commitment to care for radiotherapy patients. The cover shift will then no longer be required.

Figure 2 shows a typical three weeks of the partial shift system, adapted to accommodate the reduction in house surgeons and the loss of the cover shift from 1 August. The figure illustrates the following features: (a) night duties every seven weeks; $(b)$ daytime and weekend on call shifts with the person's own firm; (c) normal daytime shifts; (d) a maximum continuous duty of under 16 hours, except when two shifts are worked consecutively at weekends; $(e)$ a minimum of eight hours off duty between shifts; $(f)$ a minimum of two continuous periods off duty of 62 hours and 48 hours in every 28 days; $(g)$ an average working week of 64 hours, including an allowance for handovers and prospective cover.

Evaluation of revised system-A formal record of workload was kept during the first four split weekends and during the cover shift evening hours. The views of consultant and junior staff were again obtained by individual consultation and questionnaire at three and six months (at the end of house surgeons' attachments). Attention was focused on: $(a)$ the correlation between hours actually worked and contracted hours; $(b)$ the success of the attempt to link on call shifts to the firm on call; $(c)$ split weekend duties; $(d)$ night duties; $(e)$ the cover shift; $(f)$ consultants' overall assessment; $(g)$ house surgeons' overall assessment.

\section{Results}

HOURS WORKED

Reassuringly, there was a very good correlation between the hours actually worked and the contracted hours. It was exceptional for any doctor to have to work 
beyond the end of a shift. This was partially due to coordination of leave and non-abuse of prospective cover. For instance, house surgeons could not go on leave when on weekend duty unless they exchanged such duty with colleagues.

The primary aim of a reduction in average working hours to 64 hours a week, including prospective cover, was achieved and without perceived detriment to patient care or educational standards.

ON CALL SHIFTS

With the aid of our graphical display it proved easy in the revised shift system to link daytime on call shifts and weekend shifts to a house surgeon of the firm on call. All staff felt this to be a major improvement over the pilot scheme. It contributed to the job satisfaction of individual house surgeons and improved the continuity of care of patients.

\section{WEEKEND DUTIES}

The 24 hour split weekend duties proved extremely popular with all the house surgeons. Monitoring the workload over the first month proved that it was not too onerous for one house surgeon, especially as all routine weekend admissions are clerked at a preadmission clinic the week before. It was therefore unnecessary to revert to the previous system of having two house surgeons on duty simultaneously.

\section{NIGHT DUTIES}

The close monitoring over the three months of the pilot scheme had revealed that the "second on call" house surgeon was never recalled to duty after midnight. This showed that it was not necessary to have two doctors on duty overnight, nor did they have to work a second week of second on call night duty. This led to the revised scheme being based on each house surgeon working one week of night duty every eight weeks, with a more efficient cover shift replacing the second week.

\section{COVER}

Monitoring the cover shift workload showed that the evening commitment to cover radiotherapy patients usually accounted for the bulk of duties. The timing of the shift itself, while accepted as necessary, was generally unpopular among the house surgeons, who missed out on continuity of care of their firm's patients, since the heavy morning workload was perforce devolved to the other daytime staff. Fortuitously, the imminent withdrawal of a house officer post and radiotherapy commitments means that there will no longer be a requirement for this shift.

\section{CONSULTANTS' VIEWS}

The concern of the consultants was to ensure that the previous quality of patient care was maintained. There was considerable reluctance to accept the degree of crosscover required by the initial partial shift system, especially by consultants previously allocated a single house officer. That daytime and weekend duty doctors were frequently not of the same firm as that on call created problems, and consultants were able to cite instances when sick patients were not dealt with expeditiously. This was resolved by ensuring that, apart from overnight, house surgeons were always on call with their own firm, that a formal handover occurred between shifts, and that a specific doctor was delegated to cover the daytime work normally performed by the doctor on night duty. With these solutions in place the consultants accepted that partial shifts were the best way to reduce junior doctors' excessive hours of work.

Most firms have written protocols for patient management after specific surgical procedures-for example, renal transplantation, parathyroidectomy, carotid artery surgery. It was necessary to ensure that all house surgeons were familiar with these protocols, and this was an opportunity for positive educational input. Once all house surgeons were clear about their role on the night shift this aspect of the new arrangements proved particularly beneficial. Having a doctor up and about at night is a major bonus in dealing promptly with pain relief, intravenous infusions, and other problems in postoperative patients.

HOUSE SURGEONS' VIEW'S

All eight house surgeons who participated in the pilot scheme said that the biggest drawbacks were the consecutive two weeks of night duty and the long hours on call at weekends. Both these defects were corrected in the revised system.

The 16 house surgeons who completed their stints on the revised system were equally divided in their views as to which system, on call or partial shifts, was preferable. All appreciated the considerable benefits of (a) greatly reduced hours; (b) much shorter periods of continuous duty; $(c)$ better quality off duty hours, no chronic fatigue, and enhanced family and social life; (d) short weekend duties.

Their main reservations were regarding cover shifts (as detailed above) and night shifts. All said that the abolition of the cover shift would be a distinct improvement. The perceived disadvantages of night shifts were (a) temporary disruption of social life; (b) detraction of the team spirit of their firm; (c) the lack of involvement in continuity of care.

As a result seven of the 16 house surgeons said they would prefer to work an on call rota instead. On the other hand, three said they would be happy with either system, and the other six said that the overall benefits greatly outweighed the disadvantages of the night shift and that they much preferred to work a partial shift system.

\section{Discussion}

Both junior and senior doctors, ${ }^{5 \circ}$ and judges in the Court of Appeal, ${ }^{7}$ have expressed concern about excessive hours of work, and shift systems certainly provide a remedy. The introduction of a partial shift system must be seen as a dynamic process that requires regular modification. Frequent consultation with everyone involved and a willingness critically to examine existing working practices are the key elements for success.

The night shift is the main element which distinguishes a partial shift system from an on call rota. For the system to succeed there must be sufficient staff available, apart from the doctor on night duty, adequately to cover both the daytime workload and the duties of any colleague on leave.

The scheme we have illustrated is not necessarily dependent on a firm structure. However, we have shown how easily it can link in with a firm's existing on call rota by day and weekend. It is equally applicable to a self contained "specialty team" with the participation of a minimum of four junior doctors, who do not require to be all of the same grade. ${ }^{8}$ Partial shifts are not recommended for doctors in higher training, where continuity of care is more demanding and important.

Although developed in a teaching hospital with a relatively large number of junior staff in one specialty, our model can also be introduced in district general hospitals across related specialties (for example, general surgery, orthopaedics, urology, and otolaryngology). The only stipulation is that there must be a minimum of two junior doctors per firm or specialty to ensure continuity of care when one doctor is on night duty or on leave. We recommend that a partial shift 
system should comprise at least six doctors to minimise the disadvantages of night shifts.

We have also helped devise a partial shift system to be instituted from 1 August 1992 for the six house officers in the general surgical department at Guy's Hospital, by using the WordPerfect macros illustrated in this article.

Forward planning and leave coordination are essential. This is where our graphical display of the rota for six months in advance has already proved its worth, and thus the house surgeons for the next six months already have their individualised rotas, sent to them before commencing on 1 August.

In order to help individuals and hospitals efficiently to introduce and run partial shift systems we are making available the illustrated macros and tables, with more examples, and a users' guide through the Junior Doctors' Committee of the BMA (9 cm disks only). These may readily be adapted for local use. The same computer macros have also been used very successfully to design and illustrate on call rotas in general surgery and orthopaedics at St Bartholomew's Hospital.

WordPerfect 5.1 running under DOS is a prerequisite: we recommend a 286 (or faster) based PC with a laser printer. The disks can be obtained from Christine Finlan, Secretary, Junior Doctors Committee, BMA House, Tavistock Square, London WC1H 9JR.

1 Department of Health and Social Security. Funior doctors. The new deal. London: DHSS, 199

2 Nasmyth DG, Pickersgill A, Hogarth $M$. Reducing hours of work of preregistration house officers: report on a shift system. BMF 1991;302:93-4.

3 Review Body on Doctors' and Dentists' Remuneration. Tzventy-second repor. London: HMSO, 1992. (Cmnd 1813.)

4 Beecham L. Review body reports. BMF 1992;304:403,449.

5 Dillner L. Junior doctors on the warpath. BMf 1992;304:270.

6 Ross APJ Junior doctors on the warpath BM7 1992,304:385.

7 Korgaonkar G, Tribe D. Doctors' working hours and the law. Br 7 Hosp Med 1992;47:691.

8 British Medical Association. Junior doctors. The new deal. London: BMA, 1991

(Accepted 1 September 1992)

\section{Instructions to authors}

General points

- All material submitted for publication is assumed to be submitted exclusively to the $B M F$ unless the contrary is stated and should conform to the uniform requirements for manuscripts submitted to biomedical journals (the Vancouver style; BM7 1991;302:338-41).

- All authors must give signed consent to publication.

- The editor retains the customary right to style and if necessary shorten material accepted for publication.

- Type all manuscripts (including letters and obituaries) in double spacing with $3 \mathrm{~cm}$ margins.

- Number the pages.

- Give the name and address of the author to whom correspondence and proofs should be sent.

- Do not use abbreviations.

- Express all scientific measurements (except blood pressure) in SI units.

- Keep one copy of the manuscript for reference.

\section{Points specific to each section}

PAPERS, GENERAL PRACTICE, EDUCATION \& DFBATE

Papers report original research relevant to clinical medicine. They are usually up to 2000 words long with up to six tables or illustrations (short reports are up to 600 words with a maximum of one table or illustration and five references).

General Practice covers matters relevant to primary care.

Education \& Debate includes reports (up to 2000 words) on the organisation or assessment of medical work and on sociological aspects of medicine or the organisation, financing, and staffing of health services.

- Give the authors' names and initials and their posts when they did the work.

- Papers and General Practice articles should conform to the conventional format of structured abstract (maximum 250 words), introduction, methods, results, discussion, and references.

- Include a paragraph (maximum 150 words) for the This Week in $\mathrm{BMJ}$ page.

- Send three copies (if the paper is rejected these will not be returned; after three months they will be shredded).

- Whenever possible give numbers of patients or subjects studied (not percentages alone)

- Any article may be submitted to outside peer review and assessment by the editorial committee as well as statistical assessment; this takes about eight weeks.

- Manuscripts are usually published within three months of the date of final acceptance.

FFT'TERS

- Should normally be a maximum of 400 words and 10 references.

- Must be signed by all the authors.

- Preference is given to those that take up points made in articles published in the journal.

- Authors do not receive proofs.

MATERIA NON MEDICA

- Should be a maximum of 400 words.

- Authors do not receive proofs.

MEDICINE AND THE MFDIA

- Authors should discuss a proposed contribution with one of the editors before submitting it.

- Authors do not receive proofs.

PERSONAL VIEW

- Should be a maximum of 1200 words
OBITUARIES

- Should normally be a maximum of 250 words.

- Authors should summarise the person's career in a separate paragraph and not repeat these details in the main text.

- Authors do not receive proofs.

Tables

- Should be on separate sheets of paper from the text.

- Should not duplicate information given in the text of the article.

- Whenever possible, when relevant, numbers of patients or subjects studied should be given (not percentages alone)

- If a table has been published previously written consent to republication must be obtained from the copyright holder (usually the publisher) and the author(s).

Figures

- Should be used only when data cannot be expressed clearly in any other way.

- Should not duplicate information given in the text of the article.

- The numerical data on which graphs, scattergrams, and histograms are based should be supplied.

- Whenever possible, when relevant, numbers of patients or subjects studied should be given (not percentages alone).

- Legends should be on separate sheets of paper from the text.

- If a figure has been published previously written consent to republication must be obtained from the copyright holder (usually the publisher) and the author(s).

IINE DRAWINGS

- Should be presented clearly to aid redrawing.

FIGURES THAT ARE NOT LINE DRAWINGS

- Should usually be glossy prints.

- Should be no larger than $30 \times 21 \mathrm{~cm}$ (A4)

- Important areas should be indicated on an overlay.

- The top should be marked on the back.

- Photomicrographs should include an internal scale marker.

- Labelling should be on copies, not on the prints.

- Patients shown in photographs should have their identity concealed or give written consent to publication (BMF 1991;302:1194).

- Staining techniques for photomicrographs should be stated in the legend.

References

- Should be numbered in the order in which they appear in the text.

- Should give the names and initials of all the authors (unless there are more than six, when the first six should be given followed by et al); the title of the article or chapter; and the title of the journal (abbreviated according to the style of Index Medicus), year of publication, volume number, and first and last page numbers; or the names of any editors of the book, title of the book, place of publication, publisher, and year of publication, and first and last pages of the article.

- Information from manuscripts not yet in press, papers reported at meetings, or personal communications should be cited in the text, not as formal references.

Proofs and reprints

- Corrections to proofs should be kept to a minimum and should conform to the conventions shown in Whitaker's Almanack.

- If corrections need justification give this in a letter, not on the proof

- Reprints are available; a scale of charges is included when a proof is sent. 